\title{
Evaluación histopatológica del intestino de jabutis (Chelonoidis carbonarius y Chelonoidis denticulatus) criados en cautiverio y parasitados por helmintos (Chapiniella varibilis y Atractis thapari)
}

\author{
Histopathological evaluation of the intestine of jabutis (Chelonoidis carbonarius e \\ Chelonoidis denticulatus) reared in captivity and parasitized by helminths \\ (Chapiniella variabilis and Atractis thapari)

\begin{abstract}
Simone Mousinho Freire ${ }^{1,2}$, Anangela Ravena da Silva Leal ${ }^{1}$, Joilson Ferreira Batista ${ }^{1}$, Fernanda Samara Barbosa Rocha ${ }^{1}$, Silvia de Araújo França Baêta ${ }^{1}$, Ivete Lopes de Mendonça ${ }^{1}$
\end{abstract}

\section{Resumen}

\begin{abstract}
El estudio tuvo como objetivo identificar y describir lesiones intestinales ocasionadas por helmintos en jabutis (Chelonoidis carbonarius y Chelonoidis denticulatus) criados en cautiverio en el Parque Zoobotánico de Teresina, estado de Piauí, Brasil. La evaluación parasitológica de 142 de estas tortugas reveló que todas estaban parasitadas por helmintos (ascarídeos y estrongilideos), que fueron identificados por medio de microscopio de luz y microscopio electrónico como Atractis thapari y Chapiniella variabilis. Los 12 animales más parasitados (seis de cada especie) fueron eutanasiados y muestras de intestino delgado y grueso fueron evaluadas por histopatología. Macroscópicamente, todos los intestinos, tanto delgado como grueso, presentaron hiperemia. Microscópicamente, en el intestino delgado se observó hiperemia (12/12), hiperplasia difusa de células caliciformes (8/12) y edema de submucosa (1/12). En el intestino grueso se observó hiperemia, variando entre discreta a intensa $(12 / 12)$ y enteritis transmural granulomatosa focalmente extensa e intensa $(1 / 12)$.
\end{abstract}

Palabras clave: lesiones intestinales; quelonios; cautiverio

\footnotetext{
${ }^{1}$ Laboratório de Sanidade Animal, Centro de Ciências Agrárias, Universidade Federal do Piaui Campus Universitário Ministro Petrônio Portella, Teresina, Brasil

${ }^{2}$ Email: simoneuespi@gmail.com
} 
The aim of this study was to identify and describe intestinal lesions caused by helminths in red-footed tortoise (Chelonoidis carbonarius) and yellow-footed tortoise (Chelonoidis denticulatus) reared in captivity in the Zoo and Botanical Park of Teresina, Piauí State, Brazil. The parasitological evaluation of 142 of these tortoises revealed that all were parasitized by helminths (ascarids and strongyles), which were identified by light and electron microscopy as Atractis thapari and Chapiniella variabilis. The 12 most parasitized tortoises (six per species) were euthanized, and samples of small and large intestine were evaluated by histopathology. Macroscopically, all small and large intestines had hyperaemia. Microscopically, hyperaemia (12/12), diffuse goblet cell hyperplasia (8/ $12)$ and submucosa oedema $(1 / 12)$ were observed in the small intestine. Hyperaemia was observed in the large intestine, varying from discrete to intense (12/12) and focally extensive and severe transmural granulomatous enteritis (1/12).

Key words: intestinal lesions; chelonia; captivity

\section{INTRODUCCIÓN}

Los jabutis son quelonios de hábitos terrestres, pertenecientes a la familia Testudinidae, la segunda mayor familia del orden Testudines en número de especies (Ernst y Barbour, 1989). Las especies de jabutis existentes en Brasil son Chelonoidis carbonarius y Chelonoidis denticulatus (Costa y Bénils, 2014). Aunque son animales considerados silvestres y cuyo comercio es ilegal, es común la tenencia de estas especies en los hogares como mascotas. Chelonoidis denticulatus se muestra en el apéndice II de CITIES como vulnerable por la Unión Mundial de Conservación (IUCN, 2016).

Muchos nematodos se han descrito en la literatura mundial parasitando reptiles (Jacobson, 2007); sin embargo, hay pocas revisiones en la literatura, especialmente de jabutis. La mayoría de los trabajos sobre parasitología de quelonios se relaciona con especies de tortugas marinas, principalmente Chelonia mydas (Wernek y Silva, 2015; Gomes et al., 2017). Los nematodos más comunes reportados en quelonios terrestres son oxiurídeos, ascaridos, anquilostomídeos y estrongilídeos (Hedley et al., 2013). Algunos helmintos reportados en la literatura son Labiduris gulosa, Labiduris zschokkei, Labiduris irineuta, Atractis marquezi, Atractis thapari, Atractis morinae, Klossinemella travassosi, Sauricola sauricola, Chapiniella variabilis, Angusticaecum holopterum, Ophidascaris arndti, Angusticaecum brevispiculum, Helicotrema spirale, Labiduris gulosa, Labiduris irineuta, Ophiotaenia lopesi y Sauricola sauricola (Bouamer y Morand, 2006; Muniz-Pereira et al., 2009; Julca et al., 2014).

El parasitismo en los reptiles es mayormente asintomático. Solo cuando esta relación parásito-huésped se quiebra, como en el caso de altas cargas parasitarias, es que se llegan a manifestar signos clínicos (Keymer, 1978; Rose, 2005; Martínez, 2007; Hedley et al., 2013). Se han reportado casos de ulceraciones, colitis verminótica, engrosamiento de la mucosa del ciego y colon, focos hemorrágicos y congestión ocasionados por la presencia de ascarídeos y oxiurídeos en el intestino grueso de quelonios terrestres (Rideout et al., 1987; Frye, 1991; Julca et al., 2014). 
La investigación de parásitos en animales silvestres es una herramienta útil para el estudio sanitario de una población y de la calidad del ambiente, que permite comprender determinadas relaciones entre los parásitos, el huésped y el medio ambiente en que viven (Almonsay y Santos, 2002). En este trabajo se tuvo como objetivo identificar los parásitos gastrointestinales y describir las lesiones histopatológicas que ocasionan en el intestino de $C$. carbonarius y $C$. denticulatus mantenidos en cautiverio.

\section{Materiales y Métodos}

\section{Aspectos Éticos}

El estudio fue aprobado por el Comité de Ética en Experimentación Animal (CEEA) de la Universidad Federal de Piauí y del Sistema de Autorización e Información en Biodiversidad (SISBIO) a través de los opiniones 093/14 y 44782-1, respectivamente, estando en conformidad con las normas del Colegio Brasileño de Experimentación Animal (COBEA).

\section{Origen de los Animales}

Los jabutis provinieron del Parque Zoobotánico de Teresina, estado de Piauí, Brasil ( $5^{\circ} 04^{\prime} 10^{\prime \prime} \mathrm{S}$ de latitud y $42^{\circ} 76^{\prime} 87^{\prime \prime} \mathrm{W}$ de longitud). Se evaluaron 142 jabutis (40 C. Denticulatus y 102 C. carbonarius) entre junio de 2014 y junio de 2015. El recinto donde se localizan los animales en cautiverio posee un espacio totalmente abierto con una superficie total de $225 \mathrm{~m}^{2}$. En este espacio, los jabutis-tinga ( $C$. denticulatus) dividen espacio con jabutis-piranga $(C$. carbonarius). Estas instalaciones poseen un comedero, bebedero y un área libre cubierta.

\section{Análisis Parasitológico}

Para facilitar la recolección de las heces, los animales fueron colocados en decúbito dorsal en cuencas de plástico de 44x64 $\mathrm{cm}$ y $18 \mathrm{~cm}$ de profundidad, con restricción de espacio. Por tratarse de una posición incómoda, el animal defeca en respuesta al estímulo de estrés y facilita la toma de la muestra fecal.

El análisis de las muestras se hizo en el Laboratorio de Sanidad Animal del Centro de Ciencias Agrarias de la Universidad Federal de Piauí. Las muestras fueron analizadas mediante los métodos de Hoffman (1987), la técnica de Willis-Mollay (1921) modificada utilizando solución de sacarosa y la técnica de exámenes parasitológicos cuantitativos OPG según Gordon y Whitlok (1939). Se utilizó solo una muestra por animal

\section{Eutanasia de los Animales}

Los 12 jabutis más pararasitados (seis de cada especie), determinados por cualquiera de los métodos coproparasitológicos, fueron sacrificados. Para esto, se utilizó una asociación anestésica de ketamina $(15 \mathrm{mg} / \mathrm{kg})$ (Vetnil) y xilazina $2 \%(10 \mathrm{mg} / \mathrm{kg})$ (Agener União), por vía intramuscular. La muerte fue inducida luego de 45 minutos con sobredosis de tiopental sódico $\left(25 \mathrm{mg} / \mathrm{kg}\right.$ ) (Thiopentax ${ }^{\circledR}$, Cristália) por vía endovenosa (vena braquial).

Durante la necropsia y retirada del plastrón se realizó una evaluación macroscópica en busca de helmintos y anormalidades sugestivas de parasitismo en la superficie de los órganos, en el tejido muscular esquelético, en el tejido subcutáneo y en la propia cavidad celómica. Los órganos fueron separados individualmente en placas de petri conteniendo solución salina $0.9 \%$ de $\mathrm{NaCl}$ y examinados en microscopio estereoscópico.

\section{Identificación de los Parásitos}

Los helmintos fueron lavados en solución salina. Una parte de ellos fueron fijados con alcohol 70\% caliente, según protocolo de Amato et al. (1991) y otra parte fueron fijados en AFA ( $2 \%$ ácido acético glacial, 3\% de formaldehído y $95 \%$ de etanol $70^{\circ} \mathrm{GL}$ ). En 
seguida los helmintos fueron deshidratados en serie alcohólica, clarificados con lactofenol de Aman y montados en láminas temporales para análisis en microscopio de luz.

La identificación de las especies de helmintos se basó en datos morfológicos y morfométricos como longitud y anchura del cuerpo, posición del poro excretor y papila cervical, diámetro y profundidad de la cápsula bucal, número de elementos foliares y dimensiones del esófago, espirito, la cola, cantidad y disposición de las papilas caudales, usando para ello las llaves de identificación de nematodos de Yamaguti (1961), Anderson et al. (2009), Vicente et al. (1993), Skrjabin et al. (1969a, b) y artículos con descripciones específicas de especies.

Se utilizó un microscopio de luz con sistema de análisis de imágenes Leica Qwin D$1000, v, 4.1$. Para la microscopía electrónica de barrido (MEV), los helmintos se fijaron en $2.5 \%$ glutaraldehído con $0.1 \mathrm{~m}$ tampón cacodilato de sodio ( $\mathrm{pH}$ 7.4), y luego fijados en $1 \%$ tetróxido de osmio, deshidratados en serie alcohólica, secos en punto crítico con $\mathrm{CO}_{2}$ montadas sobre bases, revestidos de oro. Las láminas fueron examinadas en el microscopio electrónico de exploración VEGA-3 (Tescan) del Laboratorio de Ultraestructura / Laboratorio de Histología y Embriología animal / Instituto de Salud y Producción Animal / Universidad Federal Rural de la Amazonia (UFRA).

\section{Carga Parasitaria}

El contenido de los tractos gastrointestinales fue lavado con $500 \mathrm{ml}$ de solución fisiológica al $0.9 \%$. Se retiró $1 \mathrm{ml}$ de esta solución para el conteo de los parásitos. La carga parasitaria total fue calculada multiplicando el número de individuos por $\mathrm{ml}$ y luego multiplicado por el volumen total del conjunto. La carga parasitaria fue clasificada según Julca et al. (2014), donde la cantidad de helmintos presentes se establece como mínima (1-100), moderada (100-500) y abundante (mayor a 500).

\section{Análisis Histopatológico}

La necropsia se hizo inmediatamente después de la eutanasia. Se tomaron fragmentos del intestino delgado y del intestino grueso que presentaban las zonas más hiperémicas (Welsch y Sobotta, 2009). El tejido fue fijado en formol a $10 \%$ tamponado con fosfato 0.01M pH 7.4 (Mikel, 1994). Los tejidos fueron procesados por la técnica rutinaria de inclusiones en parafina, cortadas a $5 \mu \mathrm{m}$ y coloreados con Hematoxilina-Eosina (H-E) (Luna, 1968) para examen en microscopio de luz. Los resultados fueron presentados en forma descriptiva, estableciendo la localización (mucosa, submucosa, muscular, serosa y transmural), distribución (focal, multifocal y difusa) e intensidad (normal, mínima, moderada e intensa) de la lesión.

\section{Resultados}

\section{Infección y Carga Parasitaria}

Los 142 jabutis del estudio presentaron huevos de helmintos (Atractis thapari, Chapiniella variabilis) en al menos una de las pruebas parasitológicas realizadas (Figura 1). De los métodos utilizados, la técnica de Willis fue la que presentó los mejores resultados para determinar la presencia de huevos.

Los 12 jabutis necropsiados presentaron parásitos en el intestino grueso y cuatro $(33.3 \%)$ en el intestino delgado. No se encontraron parásitos en otros órganos. Los helmintos identificados en la necropsia fueron Atractis thapari (Petter, 1966) y Chapiniella variabilis (Chapin, 1924), habiéndose encontrado el primero en ambos intestinos. Chapiniella variabilis se encontró solo en el intestino grueso.

La carga parasitaria de $A$. thapari en el intestino grueso fue abundante, variando entre 5500 a 588500 (media: 124152 parásitos) y entre 0 y 7500 parásitos en el intestino delgado (media: 1541 parásitos). La car- 

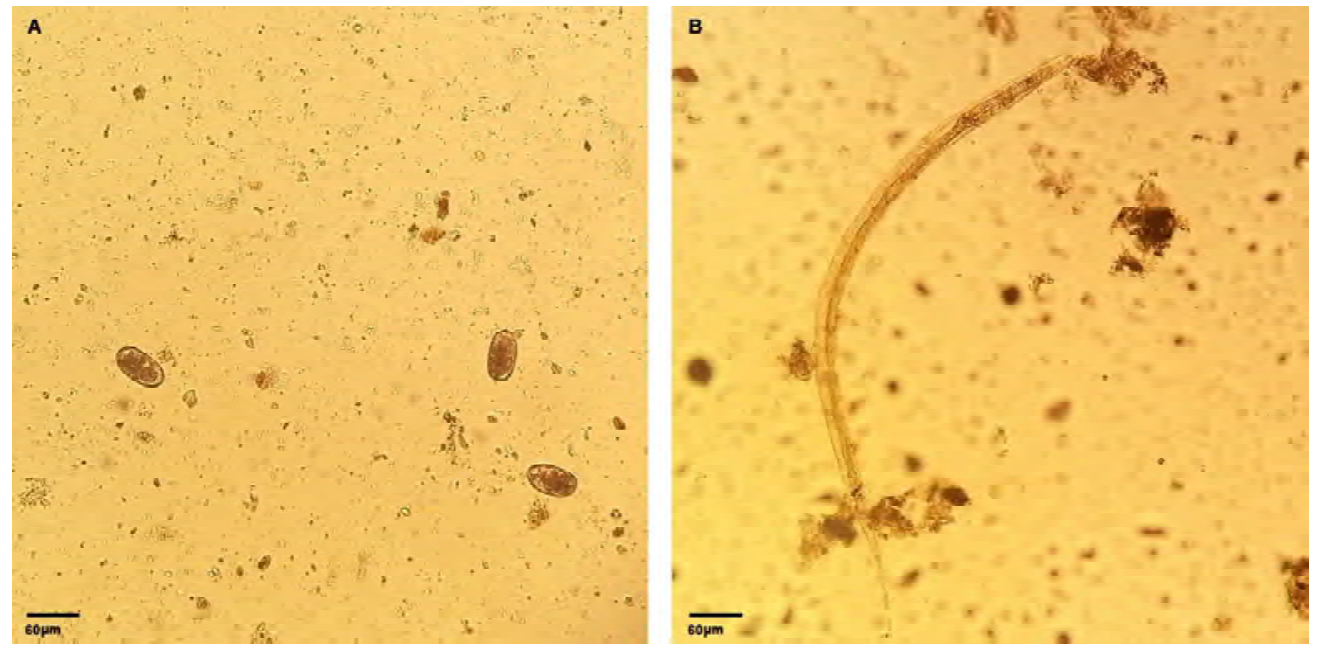

Figura 1. Huevos de helmintos en jabutis criados en cautiverio. Microscopía de luz. (A) huevos de Chapiniella variabilis. (B) larva de Atractis thapari. Escala de las barras: $60 \mu \mathrm{m}$
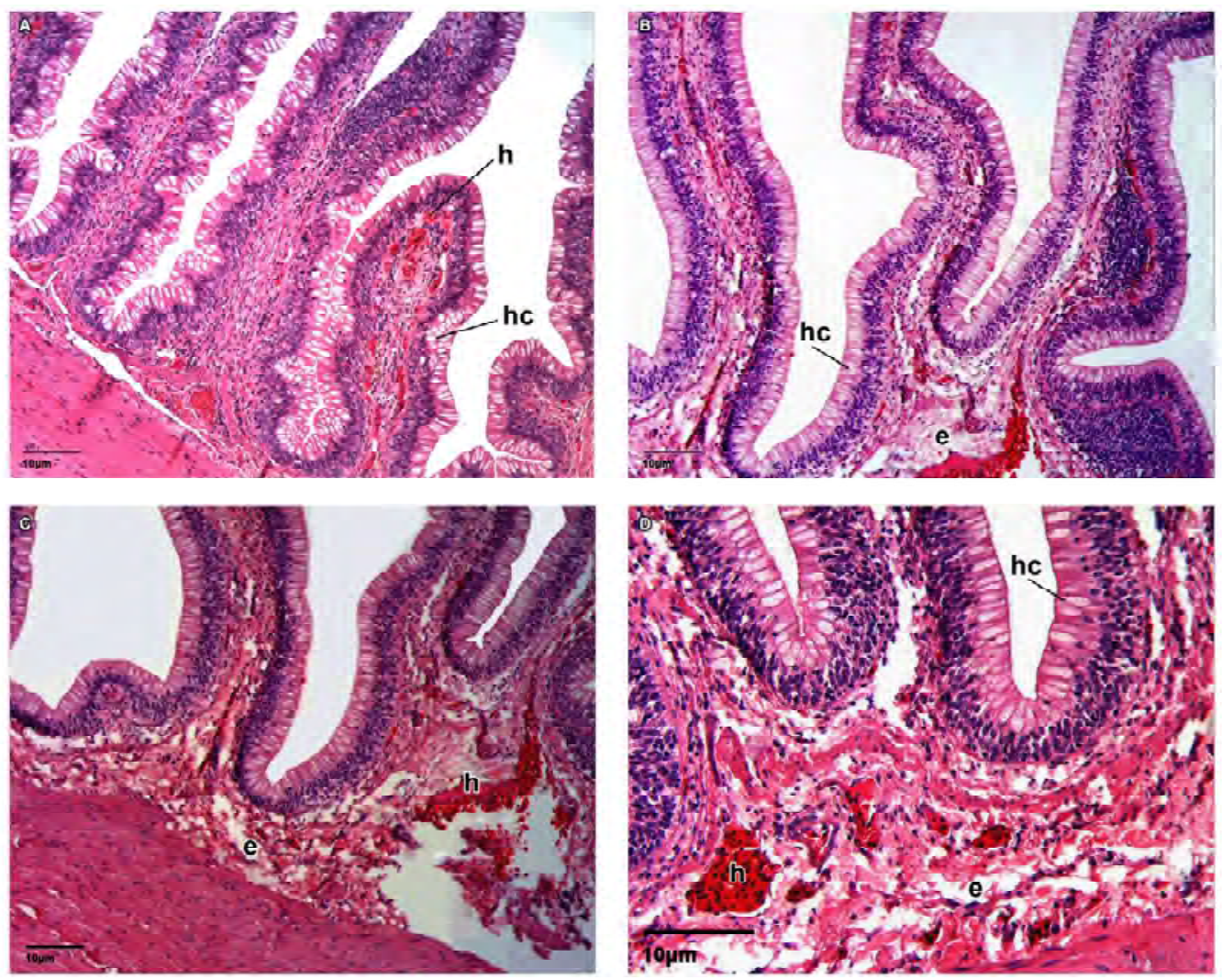

Figura 2. Intestino delgado de jabuti. A) Hiperemia (h) e hiperplasia de células caliciformes (hc), HE. B) hiperplasia de células caliciformes (hc) y edema de submucosa (e), HE. C) hiperemia (h), HE. D) hiperemia (h), hiperplasia de células caliciformes (hc) y edema de submucosa (e) HE. Escala de la barrra: $10 \mu \mathrm{m}$ 

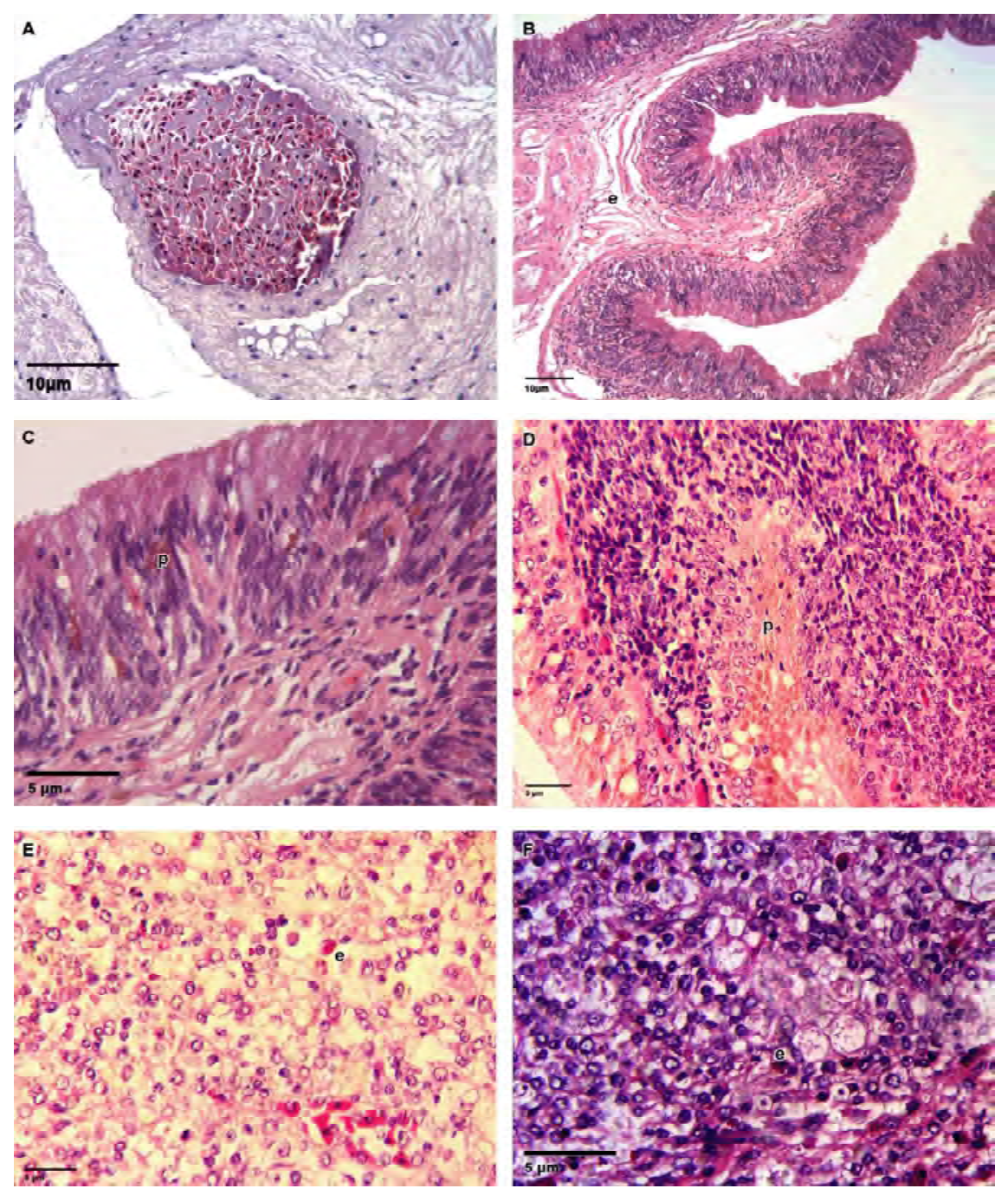

Figura 3. Intestino grueso de jabuti. A) Hiperemia, HE, escala de la barra: $10 \mu \mathrm{m}$. B) edema de submucosa (e), HE, escala de la barra: $10 \mu \mathrm{m}$. C-D) acumulación intracitoplasmática de pigmento granular marrón cobrizo (p), HE, escala de la barra: $5 \mu \mathrm{m}$. E-F) enteritis transmural mostrando infiltrado eosinofílico (e) HE, escala de la barra: $5 \mu \mathrm{m}$

ga parasitaria de Chapiniella variabilis en el intestino grueso fue abundante, variando entre 333 y 1500 (media: 652.7).

\section{Hallazgos Anatomopatológicos}

Todas las muestras (12/12) de intestino delgado presentaron alteraciones durante la evaluación macroscópica, incluyendo hiperemia (12/12) y edema de pared (1/12), contenido pastoso de coloración amarillenta (1/12), mucosa irregular (12/12) y nódulos milimétricos suaves y blanquecinos (1/12).
La evaluación histopatológica del intestino delgado reveló alteraciones en todos los animales, incluyendo hiperemia difusa, entre moderada (8/12) a intensa (4/12), hiperplasia de células caliciformes difusa entre discreta (4/12), moderada (6/12) e intensa (2/12), edema de submucosa difusa moderada, infiltrado inflamatorio linfocítico en la submucosa superficial focal moderada y áreas focales de hemorragia (1/12). Se observó una acumulación intracitoplasmática de pigmento granular marrón cobrizo en áreas focales de enterocitos (1/12) y macrófagos (1/12) en cantidad moderada (Figura 2). 
En el intestino grueso se observó hiperemia entre discreta $(3 / 12)$ a moderada $(5 / 12)$ e intensa (1/12) (los tres animales restantes no tenían hiperemia), enteritis transmural granulomatosa focalmente extensa e intensa (1/12), edema difuso moderado de submucosa ( $1 / 12)$, edema difuso discreto de submucosa (2/12), discreto infiltrado inflamatorio eosinofílico en la submucosa focal $(2 / 12)$. En el intestino grueso también se observó una acumulación intracitoplasmática de pigmento granular marrón cobrizo en áreas focales de macrófagos (5/12) en cantidad moderada (Figura 3).

\section{Discusión}

La tenencia de reptiles como mascotas ha aumentado significativamente en los últimos años, generando la aparición de nuevos ecosistemas que deben ser investigados para evaluar los riesgos relacionados. Entre estos animales se han destacado los quelonios, que aliado al comercio ilegal, ha surgido un problema que se ha vuelto común, que es el abandono de estos animales en áreas públicas y la mezcla de animales de diferentes especies conviviendo en un mismo lugar. En este contexto de cambio ecológico que involucra animales exóticos, el hombre y agentes microbiológicos, aún se tiene un conocimiento limitado sobre sus consecuencias (Hassl y Benyr, 2003; Marenzoni, 2015).

Se cree que todos los reptiles que viven en estado silvestre presentan parásitos; sin embargo, su intensidad parasitaria es generalmente baja, diferente de lo que ocurre en cautiverio, donde la disminución del espacio y la convivencia con varios animales hacen que la concentración de los parásitos sea mucho más elevada (Telford, 1971; Klingenberg, 1993). Los parásitos encontrados en reptiles mantenidos en cautiverio (parques zoológicos, granjas, mascotas) pueden inducir efectos perjudiciales a estos animales (Greiner y Mader, 2006; Rataj et al., 2011).
La técnica de OPG fue utilizada inicialmente en este estudio para, a través de los huevos, determinar la carga parasitaria de los jabutis, pues hasta entonces no se sabía las especies de parásitos que podrían ser encontradas durante la necropsia. Esta técnica también fue utilizada por Traversa et al. (2008) a fin de determinar la carga parasitaria de Testudo hermanni, Testudo graeca y Testudo marginata en Italia.

Marenzoni et al. (2015) verificaron la presencia de huevos de ascarídeos en 3 de 38 quelonios (T. hermanni, T. marginata, $T$. graeca, $T$. horsfieldii) en cautiverio. En el presente estudio, los 142 jabutis presentaron huevos de ascarídeos y estrongilídeos. Esto ocurrió porque ambas especies ( $C$. carbonarius y $C$. denticulatus) se encontraban juntas en un terrario. Los parásitos encontrados son de ciclo directo, siendo el suelo la principal fuente de contaminación (Wilson y Carpenter, 1996; Pierezan et al., 2009; Papini et al., 2011). La introducción de quelonios de diferentes orígenes en un mismo ambiente aumenta el riesgo de nuevos agentes patógenos (Marenzoni et al., 2015); además, el ambiente de cautiverio reduce la calidad del hábitat y la disponibilidad de alimento, lo que conlleva un contenido nutricional bajo y, por consiguiente, una mayor susceptibilidad a las infecciones (Primack y Rodrigues, 2001).

Chapiniella variabilis pertenece a la subfamilia Cyatostominae y las larvas de ciatostomíneos no realizan migración por la mucosa del ciego y el colon. No obstante, en grandes cantidades pueden ocasionar diarrea acuosa asociada con inflamación grave de la membrana mucosa del ciego y colon. Las lesiones se presentan como una colitis granulomatosa y masas de larvas de ciatostomíneos quedan embebidas en la membrana de la mucosa. La mayoría de estos nematodos son inmaduros, por lo que el conteo de huevos es engañosamente bajo (Bowman, 2010). Los trabajos realizados con jabutis muestran que estos parásitos han sido observados en animales con colitis (Schumacher, 
2006). La carga parasitaria observada tanto para Atractis thapari como para Chapiniella variabilis fue abundante, lo que explica la hiperemia en el intestino delgado y grueso.

La mayor cantidad de parásitos encontrados en el intestino grueso concuerda con otros estudios (Petter, 1966; Iverson, 1982; Roca, 1999; Juca et al., 2014).

Wilson y Carpenter (1996) en una revisión sobre enfermedades causadas por endoparásitos en reptiles indican que los ascarídeos y estrongilídeos son parásitos comunes de reptiles de cautiverio, causando lesiones intestinales cuando la carga parasitaria es elevada, tales como las encontradas en el presente estudio. Los ascarídeos pueden causar lesiones como larvas, a través de la migración visceral, o como adultos al fijarse en la mucosa gastrointestinal (Jacobson, 2007). En este trabajo se observó engrosamiento de la pared intestinal, hiperplasia de células caliciformes, edema de submucosa e infiltrado linfocítico en el intestino delgado e hiperemia y enteritis transmural granulomatosa en el intestino grueso; lesiones que pueden estar relacionadas con la presencia de $A$. chabaudi (ascarídeo) y $C$. variabilis (estrongilídeo). En equinos, donde los ciatostomíneos son más estudiados, los hallazgos de necropsia muestran inflamación del colon y ciego y en casos crónicos se puede observar el engrosamiento de la mucosa. En la histopatología, puede haber una respuesta celular inflamatoria con poblaciones mixtas de células mononucleares y polimorfonucleares (Corning, 2009). En otros estudios se observó enteritis hemorrágica o fibrinosa en el ciego (Bodecek et al., 2010), enteritis no supurativa e infiltración de eosinófilos, linfocitos y macrófagos en la mucosa intestinal y submucosa, así como granulomas focales y multifocal en el intestino grueso (Oryan et al., 2013).

Chapiniella variabilis fue reportado en el Perú causando granulomas eosinofílicos en la mucosa del intestino grueso en $C$. denticulatus (Julca et al., 2014). Este ciatostomíneo penetra en la mucosa intestinal en la fase larval migrando por la submucosa o muscular donde forman nódulos $\mathrm{y}$, posteriormente, retorna a la luz intestinal (Bowman, 2010). Sin embargo, en este estudio no se encontraron parásitos en los nódulos presentes en el intestino grueso de los jabutis.

Las estructuras nodulares de coloración amarillenta han sido encontradas en esófago, intestino, estómago e hígado de serpientes parasitadas con estrongilídeos. Algunos de estos nódulos contenían nematodos enrollados y en otros solo un material espeso amarillento (Mihalca, 2007). Uno de los animales evaluados en este estudio presentaba la mucosa del intestino grueso irregular por la presencia de nódulos milimétricos, blanquecinos y firmes, pero no se encontraron nematodos en la la evaluación histopatológica.

\section{Conclusiones}

- Atractis thapari se encontró parasitando intestino delgado y grueso y Chapiniella variabilis en el intestino grueso de los jabutis (Chelonoidis carbonarius y Chelonoidis denticulatus) mantenidos en cautiverio en el Parque Zoobotánico de Teresina-Piauí.

- Las lesiones anatomopatológicas e histopatológicas observadas en el intestino grueso de jabutis (Chelonoidis carbonarius y Chelonoidis denticulatus) pueden estar relacionadas con la presencia de los helmintos Atractis thapari y Chapiniella variabilis debido a la alta carga parasitaria encontrada.

\section{Literatura Citada}

1. Almonsay NRP, Santos LC. 2002. Laboratory support in wild animal medicine. In: Fowler ME, Cubas ZS (eds). Biology, medicine and surgery of South American wild animals. Iowa, USA: Iowa State University Press. p 500-503. 
2. Amato JFR, Boeger WA, Amato SB. 1991. Protocolos para laboratório - Coleta e processamento de parasitos de pescado. Rio de Janeiro: UFRRJ. 81 p.

3. Anderson RC, Chabaud AG, Willmott S. 2009. Keys to the nematode parasites of vertebrates. Archival Volume. UK: CAB International. $480 \mathrm{p}$.

4. Bodecek P, Jahn O, Dobesova E, Vavrouchova A. 2010. Equine cyathostomosis: case reports. Vet MedCzech 55: 187-193.

5. Bouamer S, Morand S. 2006. Nematodes parasites of Testudinidae (Chelonia): list of species and biogeographical distribution. Ann Zool 56: 225240. doi: 10.3161/00034540677-8700775

6. Bowman DD. 2010. Parasitologia veterinária de Georgis. $9^{\text {th }}$ ed. Rio de Janeiro: Elsevier. 488 p.

7. Chapin EA. 1924. Nematode parasites of the Brazilian land-tortoise, Testudo denticulata. Proc US Natl Mus 65: 1-6. doi: 10.5479/si.00963801.65-2526.1

8. Corning S. 2009. Review equine cyathostomins: a review of biology, clinical significance and therapy. Parasite Vector 2(Supp12): S2. doi: 10.1186/17563305-2-S2-S1

9. Costa HC, Bérnils RS. 2014. Répteis brasileiros: lista de espécies. Herpetol Bras 3: 75-79.

10. Ernst CH, Barbour RW. 1989. Turtles of the world. Washington: Smithsonian Institution Press. $313 \mathrm{p}$.

11. Frye FL. 1991. Applied clinical nonhemic parasitology of reptiles. In: Frye FL (ed). Biomedical and surgical aspects of captive reptile husbandry. $2^{\text {th }}$ ed. Krieger, Florida: Malabar. p 281-325.

12. Gomes MC, Martins IVF, Werneck MR, Pavanelli L. 2017. Ecologia da comunidade de helmintos gastrointestinais de tartarugas-verdes (Chelonia mydas) recolhidas no litoral do Espírito Santo. Arq Bras Med Vet Zootec 69: 44650. doi: 10.1590/1678-4162-9039

13. Gordon H, Whitlock HV. 1939. A new technique four counting nematode eggs in sheep faeces. $J$ Sci Ind Res 12: 50-52.
14. Greiner EC, Mader DR. 2006. Parasitology. In: Mader DR (ed). Reptile medicine and surgery. $2^{\text {nd }}$ ed. St.Louis, US: Saunders Elsevier. p 343-364.

15. Hassl A, Benyr G. 2003. Hygienic evaluation of terraria inhabited by amphibians and reptiles: Cryptosporidia, free-living amebas, Salmonella. Wien Klin Wochenschr 115 (Suppl 3): 68-71.

16. Hedley J, Eatwell K, Shaw DJ. 2013. Gastrointestinal parasitic burdens in UK tortoises: a survey of tortoise owners and potential risk factors. Vet Rec 173: 525. doi: 10.1136/vr.101794

17. Hoffmann RP. 1987. Diagnóstico de parasitismo veterinário. Rio de Janeiro: Sulina. $156 \mathrm{p}$.

18. [IUCN] International Union for Conservation of Nature. 2016. IUCN red list of threatened species. [Internet]. Available in: https://www.iucnredlist.org/

19. Iverson JB. 1982. Adaptations to herbivore in iguanine lizards. In: Burghardt GM, Rand AS (ed). Iguanas of the world: their behavior, ecology and conservation. New York, USA: Noyes Publ. p 60-76.

20. Jacobson ER. 2007. Parasites and parasitic diseases of reptiles. In: Jacobson ER (ed). Infectious diseases and pathology of reptiles, color atlas and text. USA: CRC Press. p 571-597.

21. Julca RR, Casas EA, Chavera AC, Sánchez LP, Sánchez NP, Batalla LL. 2014. Descripción anatomopatológica de lesiones por helmintos gastrointestinales en tortugas motelo (Chelonoidis denticulata). Rev Inv Vet Perú 25: $37-$ 50. doi: 10.15381/rivep.v25i1.8466

22. Keymer IF. 1978. Diseases of chelonians: (1) Necropsy survey of tortoises. Vet Rec 103: 548-552. doi: 10.1136/ vr.103.25.548

23. Klingenberg R.J. 1993. Understanding reptile parasites: a basic manual for herpetoculturists \& veterinarians. Lakeside, CA, USA: Advanced Vivarium Systems. 81 p.

24. Luna GL. 1968. Manual of histologic staining methods of the Armed Forces Institute of Pathology. New York: McGraw Hill. 258 p. 
25. Marenzoni ML, Zicavo A, Veronesi F, Morganti G, Scuota S, Coletti M, Passamonti F, et al. 2015. Microbiological and parasitological investigation on chelonians reared in Italian facilities. Vet Ital 51: 173-178. doi: 10.12834/ VetIt.7.21.3

26. Martínez A. 2007. Parásitos digestivos en reptiles. Argos Inf Vet 88: 48-49.

27. Mihalca AD, Fictum P, Skoric M, Sloboda M, Kärvemo S, Ghira I, Carlsson M, et al. 2007. Severe granulomatous lesions in several organs from Eustrongylides larvae in a freeranging dice snake, Natrix tessellata. Vet Pathol 44: 103-105. doi: 10.1354/ vp.44-1-103

28. Mikel UV. 1994. Advanced laboratory methods in histology and pathology. Washington DC: American Registry of Pathology. 254 p.

29. Muniz-Pereira LC, Vieira FM, Luque JL. 2009. Checklist of helminth parasites of threatened vertebrate species from Brazil. Zootaxa 2123:1-45. doi: 10.5281/ zenodo. 188170

30. Oryan G, Farjani K, Rajabloo M. 2013. Larval cyathostominosis in a working donkey. J Parasit Dis 39: 324327. doi: 10.1007/s12639-013-0313-6

31. Papini R, Manetti C, Mancianti F. 2011. Coprological survey in pet reptiles in Italy. Vet Rec 169: 207. doi: $10.1136 /$ vr.d4398

32. Petter AJ. 1966. Equilibre des especes dans les populations the nematodes parasites du colon des tortues terrestres. Mem Mus Nat Hist Ser AZool 39: 1-252.

33. Pierezan F, Rissi DR, Oliveira JC, Lucena RB, Tochetto C, Flores MM, et al. 2009. Enterite granulomatosa associada a larvas de Ciatostomíneos em eqüinos no Rio Grande do Sul. Pesq Vet Bras 29: 382-386. doi: 10.1590/S0100736X2009000500004

34. Primack BR, Rodrigues E. 2001. Biologia da conservação. Brazil: Universidade Estadual de Londrina. 328 p.
35. Rataj AV, Lindtner-Knific R, Vlahovice K, Mavri U, Dovè A. 2011. Parasites in pet reptiles. Acta Vet Scand 53: 33. doi: 10.1186/1751-0147-53-33

36. Rideout BA, Montali RJ, Phillips LG, Gardiner CH. 1987. Mortality of captive tortoises due to viviparous nematodes of the genus Proatractis (Family Atractidae). J Wildlife Dis 23: 103-108.

37. Roca V. 1999. Relación entre las faunas endoparásitas de reptiles y su tipo de alimentación. Rev Esp Herp 13: 101-121.

38. Rose K. 2005. Common diseases of urban wildlife. Reptiles. The Australian Registry of Wildilife Health. [Internet]. Available in: https://arwh.org/sites/default/files/2016-11/Common\%-20Diseases\%20of\%20Reptiles_with_images.pdf

39. Schumacher J. 2006. Selected infectious diseases of wild reptiles and amphibians. J Exot Pet Med 15: 18-24. doi: 10.1053/j.jepm.2005.11.004

40. Skrjabin KI, Shikhobalova NP, Schulz RS, Popova TI, Boev SN, Delyamure SL. 1969a. Key to parasitic nematoda. Spirurata and Filariata. Jerusalem: Israel Program for Scientific Translations. $508 \mathrm{p}$.

41. Skrjabin KI, Shikhobalova NP, Schulz RS, Popova TI, Boev SN, Delyamure SL. $1969 b$. Key to parasitic nematoda. Strongylata. Jerusalem: Israel Program for Scientific Translations. $890 \mathrm{p}$.

42. Telford SR. 1971. Parasitic diseases of reptiles. J Am Vet Med Assoc 159: 16441652.

43. Traversa D, Iorio R, Otranto D, Modrý D, Slapeta J 2008. Cryptosporidium from tortoises: genetic characterisation, phylogeny and zoonotic implications. Mol Cell Probe 22: 122-128. doi: 10.1016/ j.mcp.2007.11.001

44. Vicente JJ, Rodrigues HO, Gomes DC, Pinto RM. 1993. Nematoides do Brasil, Parte III, Nematoides de répteis. Rev Bras Zool 10: 19-168. doi: 10.1590/ S0101-81751993000100003.

45. Welsch U, Sobotta J. 2009. Histología. $2^{\circ}$ ed. España: Médica Panamericana. 699 p. 
46. Willis HH. 1921. A simple levitation method for the detection of wookworm ova. Med J Australia 8: 375-376.

47. Werneck MR, Silva RJ. 2015. Parasitas de helmintos das tartarugas verdes juvenis Chelonia mydas (Testudines: Cheloniidae) no Brasil. J Parasitol 101): 713-716. doi: 10.1654/15-780
48. Wilson SC, Carpenter JW. 1996. Endoparasitic diseases of reptiles. Semin Avian Exot Pet 5: 64-74. doi: 10.1016/ S1055-937X(96)80019-3

49. Yamaguti S. 1961. Systema Helminthum. Nematodes. New York: Interscience Publishers. 1261 p. 